\section{Photo-sputtering of ice and hydrogen around Saturn's rings}

\section{R. W. Carlson}

Earth and Space Sciences Division, Jet Propulsion Laboratory, California Institute of Technology, Pasadena, California 91109

Rocketborne UV spectroscopic measurements have shown that a surprisingly dense cloud of atomic hydrogen surrounds Saturn ${ }^{1,2}$ which has been interpreted as being associated with the Saturn ring system. Verification of an UV emission cloud related to the rings is given by Pioneer 11 fiyby measurements ${ }^{3}$. Several ring sources of atomic hydrogen have been proposed ${ }^{4-7}$, but abundance and lifetime estimates given below require a source which is much more potent than those previously considered. An alternate mechanism which satisfies this source requirement is proposed here, and involves photodissociation of surface water molecules on the icy ring material.

The rocket measurements of Weiser et al. ${ }^{1,2}$ show hydrogen Ly $\alpha$ emissions outside the disk of Saturn with a mean brightness of $200 \mathrm{R} \pm 50 \%$. If this emission is produced by resonance scattering of the incident solar Lyo line with an efficiency ${ }^{8}$ of $2.3 \times 10^{-5} \mathrm{~s}^{-1}$ atom $^{-1}$, then the total number of atoms within their viewing aperture is $N \approx 8 \times 10^{33}$ atoms (assuming optically thin conditions). The mean density is $n \approx 400 \mathrm{~cm}^{-3}$. One can relate this abundance to a production rate through the hydrogen atom lifetime as discussed below.

Loss of $\mathrm{H}$ atoms can be caused by many processes, including photoionisation and charge exchange. However, the major loss of $\mathrm{H}$ atoms produced in, or near, the rings is through collisions and absorption by ring particles. Laboratory measurements ${ }^{9}$ of the reflection of $\mathrm{H}$ atoms by low temperature ice show reflection coefficients of $78 \%$, that is, $22 \%$ of the collisions will result in loss of a hydrogen atom. The low density of the gaseous ring 'atmosphere' implies that molecular collisions are infrequent and that atoms follow keplerian orbits about Saturn. The trajectories of atoms created near the rings will pass through the rings generally once per orbit, twice if the orbital eccentricity is small. As the optical thickness of the rings is about unity ${ }^{10}$, then the probability of an atom striking a ring particle is $\approx\left(1-\mathrm{e}^{-1}\right)=$ 0.63 . For most of these collisions, the atom will be reflected, but roughly one impact in five will result in loss of an atom. For a nominal keplerian orbital period of $T=4 \times 10^{4} \mathrm{~s}$, the resulting lifetime is $\tau=T /(0.63 \times 0.22)=3 \times 10^{5} \mathrm{~s}$. With this lifetime, the source rate $Q$ required to satisfy the observations is estimated to be $Q=N / \tau=8 \times 10^{33} / 3 \times 10^{5}=3 \times 10^{28}$ atom s$~^{-1}$. Note that this derived source rate is insensitive to the actual trajectories. If the orbits extend beyond the field-of-view of the rocket aperture (which was used for the abundance estimate) then the fraction of time that an atom spends within the field is less than unity, and the orbital period $T$ is also greater. As both the total abundance and lifetime estimates are increased, then the derived source rate is approximately the same.

The first investigations of $\mathrm{H}$ atom production by the rings were presented by Blamont ${ }^{4}$ and Dennefeld ${ }^{5}$, who considered

Table 1 Ring $\mathrm{H}$ atom production rates

\begin{tabular}{lc}
\hline \multicolumn{1}{c}{ Mechanism } & $\mathrm{Q}\left(\right.$ atom s$\left.^{-1}\right)$ \\
Photo-sputtering & $1.5 \times 10^{28}$ \\
Photoelectron-ion neutralisation & $9 \times 10^{23}-9 \times 10^{24}$ \\
Sublimation, dissociation & $2.2 \times 10^{24}$ \\
Meteoritic bombardment & $1.8 \times 10^{24}$ \\
Magnetospheric ion sputtering & $<6 \times 10^{23}$ \\
Solar wind & $1 \times 10^{23}$ \\
Interstellar gas & $2.2 \times 10^{22}$ \\
\hline
\end{tabular}

several sources, including sublimation and dissociation of ring material, and bombardment by meteoroids, the solar wind, and interstellar gas. Their calculated source rates are summarised in Table 1 and are evidently too small to account for the required value of $3 \times 10^{28}$ atom s ${ }^{-1}$. Cheng and Lanzerotti ${ }^{6}$ proposed sputtering by high energy ions as a ring erosion mechanism. Assuming that peak jovian fluxes can be used to describe the saturnian magnetosphere, they predicted a hydrogen atom source rate $\approx 1.2 \times 10^{27} \mathrm{~s}^{-1}$. However, the Pioneer 11 flyby has shown that the Saturn magnetosphere is much less intense than that of Jupiter, and the peak fluxes used by Cheng and Lanzerotti should be reduced by a factor of at least 20 , and probably more, based on measured saturnian proton and electron fluxes $^{11,12}$. Furthermore, proton fluxes ${ }^{12}$ in the vicinity of the rings are $<10^{-2}$ of the peak values, which results in an ionsputtering rate of $<6 \times 10^{23}$ atom $\mathrm{s}^{-1}$. An additional mechanism has been proposed by $\mathrm{Ip}^{7}$-neutralisation by ring particles of the photoelectron-ion flux flowing out from the Saturn ionosphere along magnetic field lines. The magnitude of this source is also insufficient to account for the observations (see Table 1).

Harrison and Schoen ${ }^{13}$ pointed out that UV radiation $(1,000 \leqslant \lambda \leqslant 1,800 \AA)$ will dissociate water molecules on ice surfaces and emit hydrogen atoms and hydroxyl radicals. Similar processes have been found to occur on UV irradiation of glasses and semiconductors ${ }^{14,15}$. For photo-absorption which occurs in the outer few molecular layers of an ice crystal, the probability of escape from the crystal is high. At the orbit of Saturn, the molecular photodissociation rate ${ }^{16}$ due to solar UV radiation is $1.1 \times 10^{-7} \mathrm{~s}^{-1}$ molecule ${ }^{-1}$. If we assume that only the single outermost monolayer of molecules $\left(1 \times 10^{15}\right.$ molecule $\left.\mathrm{cm}^{-2}\right)$ of the icy ring material will contribute to the flux escaping from the solid, then we find a hydrogen atom flux of $1.1 \times$ $10^{8}$ atom cm $\mathrm{cm}^{-2} \mathrm{~s}^{-1}$. Although the initial energy distribution cannot be accurately determined, most of these atoms should possess less than the $3 \mathrm{eV}$ of energy required for escape from the Saturn system, due to simultaneous vibrational and electronic excitation of $\mathrm{OH}$, collisions with other surface molecules, and subsequent collisions with ring particles.

The projected area of the $A+B$ rings at the time of the rocket measurements was $1.4 \times 10^{20} \mathrm{~cm}^{2}$, resulting in a total source rate of $Q=1.5 \times 10^{28}$ atom s $\mathrm{s}^{-1}$. This value is compared with other mechanisms in Table 1.

The source rate for the mechanism proposed here is within a factor of two of the required value and is within the experimental error of the measurement. Considering the spirit of the calculations, the agreement is quite satisfactory, arguing that the proposed mechanism is a viable explanation for the observed Ly $\alpha$ glow. It may be possible to test this, and other, source hypotheses through the recent Pioneer 11 and forthcoming Voyager flybys. For the present mechanism, the source strength during these flybys will be smaller due to the smaller inclination of the ring plane relative to the Sun.

This paper presents the results of one phase of research carried out at the Jet Propulsion Laboratory, California Institute of Technology, under contract NAS 7-100.

Received 17 August; accepted 5 December 1979.

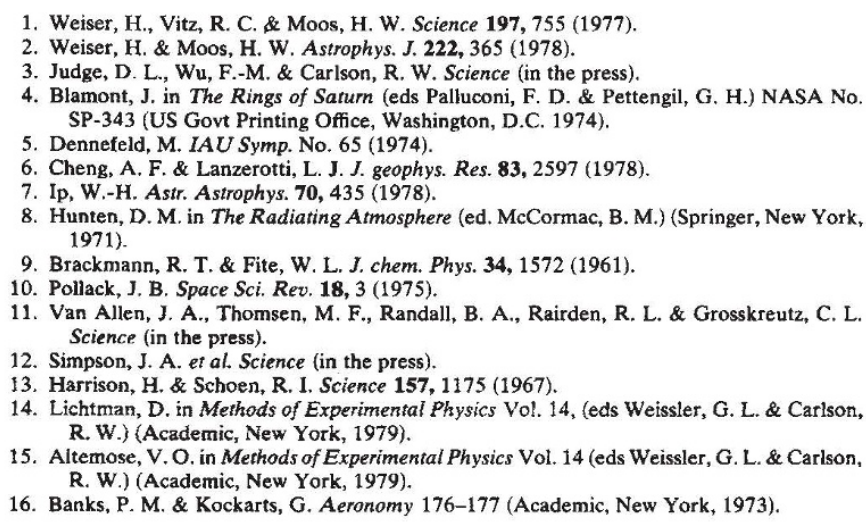

